$11-1-2019$

\title{
Investir dans le changement social et comportemental est une solution rentable pour augmenter l'utilisation des contraceptifs modernes en Guinée
}

Breakthrough RESEARCH

Follow this and additional works at: https://knowledgecommons.popcouncil.org/departments_sbsr-rh How does access to this work benefit you? Let us know!

\section{Recommended Citation}

Breakthrough RESEARCH. 2019. «Investir dans le changement social et comportemental est une solution rentable pour augmenter l'utilisation des contraceptifs modernes en Guinée », fiche d'information. Washington, DC : Population Council. 


\section{Investir dans le changement social et comportemental est une solution rentable pour augmenter l'utilisation des contraceptifs modernes en Guinée}

Les actions en matière de changement social et comportemental (CSC) sont généralement considérées comme primordiales dans l'offre de services de santé de haute qualité, mais le manque d'informations concernant le coût et l'impact du CSC signifie que les décideurs ont sous-estimé et sous-financé le CSC. Pour remédier à ce problème, Breakthrough RESEARCH a adopté une nouvelle approche afin de lier les données probantes aux décisions potentielles en matière d'investissement grâce à leur récente publication, intitulée Analyse de rentabilité pour investir dans le changement social et comportemental en matière de planification familiale qui rassemble des données provenant de 130 études sur l'impact du CSC et de 53 études sur le coût du CSC. L'ensemble de ces données probantes montre que :

\section{LE CSC EST EFFICACE POUR AUGMENTER L'UTILISATION DES CONTRACEPTIFS MODERNES.}

Des études révèlent que le CSC influence de façon positive les résultats intermédiaires qui impactent l'utilisation des contraceptifs modernes, notamment les attitudes, la communication avec les partenaires et autres. L'ampleur de l'impact varie sel- on le type d'action et le résultat intermédiaire visé (graphique 1).

\section{LES RÉSULTATS INDIQUENT QUE LE CSC EST UNE ACTION EXTRÊMEMENT ÉCONOMIQUE.}

Le coût par année de vie corrigée de l'incapacité $(A V C I)^{1}$ évitée est une mesure courante pour évaluer la rentabilité. Cette analyse a révélé que le coût par $\mathrm{AVCI}$ évitée pour le CSC en matière de planification familiale était de 468 US\$ en Égypte, 591 US\$ aux Philippines, 1051 US\$ en Zambie et 438 US\$ en Guinée. Ces résultats sont inférieurs aux seuils de l'Organisation mondiale de la santé pour être considérés comme une action extrêmement rentable. ${ }^{2}$

\section{LE CSC PEUT GÉNÉRER UN RETOUR SUR INVESTISSEMENT POSITIF.}

La simulation de l'analyse de rentabilité de Breakthrough RESEARCH en Zambie a révélé que chaque dollar investi dans I'intensification du CSC permet d'économiser entre 2,40 \$ et 5,30 \$ si l'on tient compte des coûts directs des soins de santé et des pertes de productivité. En Guinée, 1 \$ investi dans le CSC génère entre 2,30 \$ et 6,10\$ d'économies.

\section{GRAPHIQUE 1 : LE CSC AFFECTE LES VOIES D'ACCÈS VERS L'AUGMENTATION DE L'UTILISATION DES CONTRACEPTIFS MODERNES}

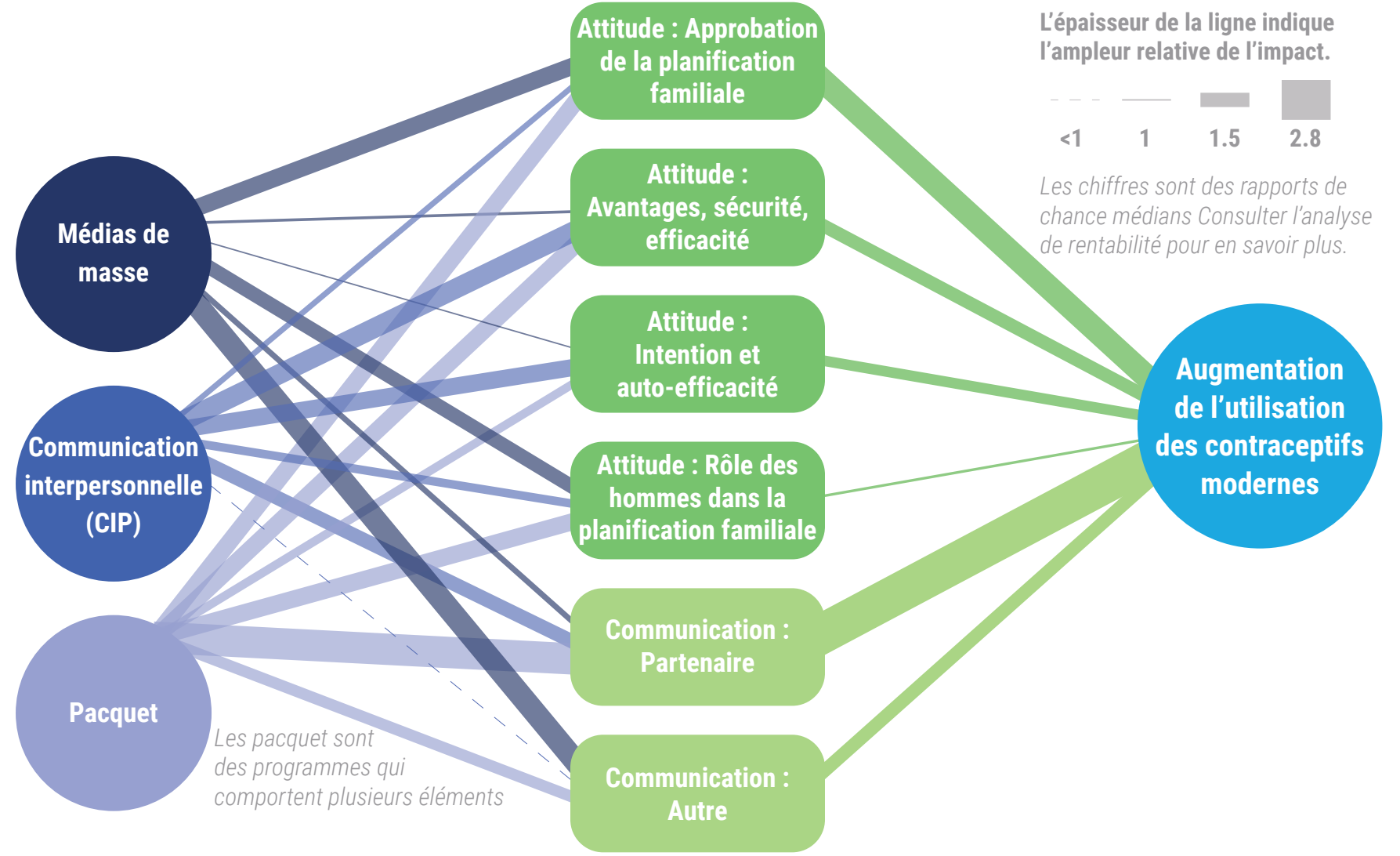




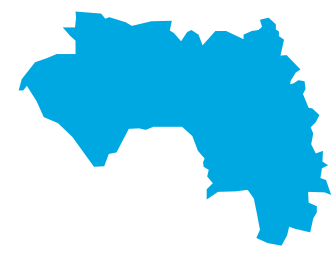

Investir dans le CSC en Guinée peut aider à accroître l'impact des investissements réalisés dans la prestation de services de planification familiale en améliorant les attitudes, les croyances et la communication, contribuant ainsi à l'augmentation de l'utilisation des contraceptifs modernes. Ces rendements, comparés aux coûts, font des interventions de CSC un investissement très rentable en Guinée.

\section{LE SBC POUR LA PLANIFICATION FAMILIALE : UN INVESTISSEMENT RENTABLE POUR LA GUINÉE}

L'analyse de rentabilité de Breakthrough RESEARCH a modélisé la rentabilité globale et le retour sur investissement du CSC en Guinée, en combinant les données sur le coût et l'efficacité du CSC et les données spécifiques à la Guinée sur son contexte actuel et l'intensification prévue du CSC alignée sur le Plan d'action national budgétisé pour la planification familiale (2019-2023).

\section{IMPACT}

Sur cinq ans (2019-2023), on estime que les investissements dans le CSC conformément aux stratégies nationales contribueront à :

- 166500 utilisateurs de contraceptifs modernes supplémentaires d'ici 2023

- Une augmentation de 3 points de pourcentage du taux de prévalence des contraceptifs modernes d'ici 2023

- 135700 grossesses non désirées évitées de façon cumulative

- $34800 \mathrm{AVCl}$ gagnées de façon cumulative

\section{GRAPHIQUE 2 : CONTRIBUTION RELATIVE DES INTERVENTIONS DU SBC À L'AUGMENTATION DE L'UTILISATION DES CONTRACEPTIFS MODERNES EN GUINÉE}

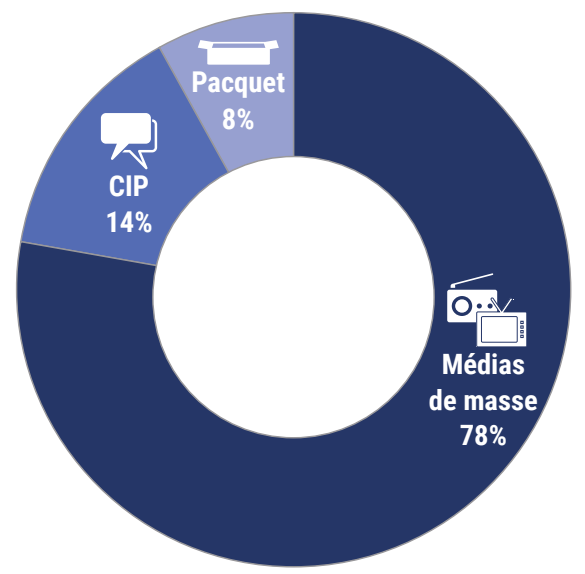

\section{COÛT}

La réalisation de ces impacts nécessiterait un investissement de cinq ans dans les actions du CSC estimé à 12,2 million de dollars. ${ }^{3}$ Un montant supplémentaire de 3 millions de dollars serait nécessaire pour couvrir les coûts directs de prestation des services liés à l'augmentation du nombre d'utilisateurs qui utilisent les services.

\section{RENTABILITÉ GLOBALE}

Le scénario d'intensification des actions du CSC est considéré comme très rentable en fonction de la norme de l'Organisation mondiale de la santé qui est de moins d'une fois le produit intérieur brut par habitant :

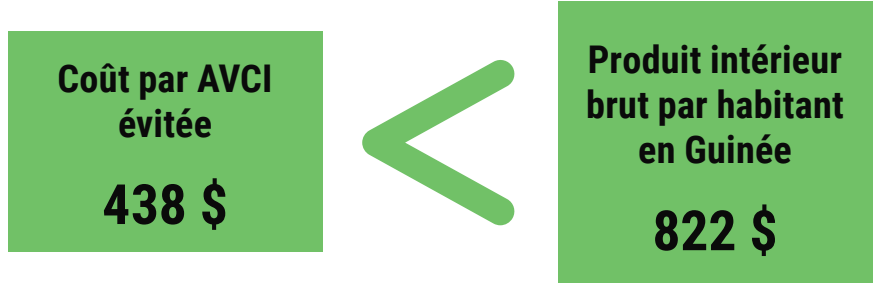

\section{RETOUR DES INVESTISSEMENTS}

Chaque dollar investi dans le CSC en Guinée peut faire gagner de 2 à 6 \$ liés aux coûts de soins directs (liés à la grossesse et aux accouchements) et aux pertes de productivité (grâce à la réduction de la mortalité et de la morbidité maternelle).

${ }^{1}$ «Une AVCl est comparable à une année perdue de vie «saine». La somme des AVC sur toute la population, ou le fardeau de la maladie, sont comparables à la mesure de l'écart entre la situation de santé actuelle et une situation idéale dans laquelle l'ensemble de la population vit jusqu'à un âge avancé, sans maladie ni handicap. »(OMS, https://www.who.int/healthinfo/glob-al_burden_disease/metrics_daly/ en/, date d'accès 29 octobre 2019)

¿Le seuil de l'Organisation mondiale de la santé pour une action extrêmement rentable est un coût par AVCl évitée inférieur à une fois le produit intérieur brut par habitant. Par rapport à d'autres interventions sanitaires, ces résultats se situent dans la moyenne des coûts par AVCl évitée (Commission Macroéconomie et Santé de l'OMS 2001 ; Horton et al. 2017).

${ }^{3}$ Coût médian estimatif de 12,2 million de \$ (fourchette: 8,2-21,0 millions de \$). Estimations de coûts basées sur les coûts unitaires mondiaux et les scénarios d'intensification supposés; les coûts réels en Guinée peuvent varier.

\section{Breakthrough RESEARCH}

Cette activité est mise en œuvre dans le cadre de Breakthrough RESEARCH. Breakthrough RESEARCH catalyse le changement social et comportemental (SBC) en menant des études et des évaluations de pointe et en favorisant des solutions fondées sur des données probantes pour améliorer les programmes de santé et de développement à travers le monde. Breakthrough RESEARCH est un consortium dirigé par Population Council en partenariat avec Avenir Health, ideas42, Institute for Reproductive Health de Georgetown University, Population Reference Bureau et Tulane University.

\section{Remerciements}

Le projet Breakthrough RESEARCH est possible grâce au généreux soutien de la population américaine par l'intermédiaire de l'Agence des États-Unis pour le développement international (USAID), selon les termes de l'accord de coopération $n^{\circ}$. AID-OAA$A A-17-00018$. Le contenu de ce document relève de la seule responsabilité de Breakthrough RESEARCH et de Population Council et ne reflète pas nécessairement les vues de I'USAID ou du gouvernement des États-Unis.

\section{Breakthrough RESEARCH}

Population Council

4301 Connecticut Avenue, NW, Suite 280

Washington, DC 20008

Tel: +1 2022379400

breakthroughactionandresearch.org

Citation suggérée : Breakthrough RESEARCH. 2019. «Investir dans le changement social et comportemental est une solution rentable pour augmenter l'utilisation des contraceptifs modernes en Guinée ", fiche d'information. Washington DC : Population Council. 